\title{
Suboptimal Iodine Status among Pregnant Women in the Oslo Area, Norway
}

\author{
Sigrun Henjum ${ }^{1, *(1)}$, Inger Aakre ${ }^{1}$, Anne Marie Lilleengen ${ }^{1}$, Lisa Garnweidner-Holme ${ }^{1}$, \\ Sandra Borthne ${ }^{1}$, Zada Pajalic ${ }^{1}$ (D), Ellen Blix ${ }^{1}$, Elin Lovise Folven Gjengedal ${ }^{2}$ \\ and Anne Lise Brantsæter ${ }^{3}$ (D) \\ 1 Department of Nursing and Health Promotion, Faculty of Health Sciences, OsloMet — Oslo Metropolitan \\ University, Oslo 0310, Norway; inger.aakre@hioa.no (I.A.); annemarie.lilleengen@hioa.no (A.M.L.); \\ lisa.garnweidner-holme@hioa.no (L.G.-H.); sborthne@gmail.com (S.B.); Zada.Pajalic@hioa.no (Z.P.); \\ Ellen.Blix@hioa.no (E.B.) \\ 2 Faculty of Environmental Sciences and Natural Resource Management, Norwegian University of Life \\ Sciences, Aas 1433, Norway; elin.gjengedal@nmbu.no \\ 3 Division of Infection Control and Environmental Health, Norwegian Institute of Public Health, \\ Oslo 0403, Norway; AnneLise.Brantsaeter@fhi.no \\ * Correspondence: sigrun.henjum@hioa.no; Tel.: +47-6723-6513
}

Received: 31 January 2018; Accepted: 22 February 2018; Published: 28 February 2018

\begin{abstract}
Norway has been considered iodine replete for decades; however, recent studies indicate reemergence of inadequate iodine status in different population groups. We assessed iodine status in pregnant women based on urinary iodine concentration (UIC), urinary iodine excretion (UIE), and iodine intake from food and supplements. In 804 pregnant women, 24-h iodine intakes from iodine-rich foods and iodine-containing supplements were calculated. In 777 women, iodine concentration was measured in spot urine samples by inductively coupled plasma/mass spectrometry (ICP-MS). In addition, 49 of the women collected a 24-h urine sample for assessment of UIE and iodine intake from food frequency questionnaire (FFQ). Median UIC was $92 \mu \mathrm{g} / \mathrm{L}$. Fifty-five percent had a calculated iodine intake below estimated average requirement (EAR) $(160 \mu \mathrm{g} /$ day). Iodine intake from food alone did not provide the amount of iodine required to meet maternal and fetal needs during pregnancy. In multiple regression models, hypothyroidism, supplemental iodine and maternal age were positively associated with UIC, while gestational age and smoking were negatively associated, explaining $11 \%$ of the variance. This study clearly shows that pregnant women in the Oslo area are mild to moderate iodine deficient and public health strategies are needed to improve and secure adequate iodine status.
\end{abstract}

Keywords: iodine status; pregnancy; iodine deficiency; iodine intake; urinary iodine concentration; urinary iodine excretion; Norway

\section{Introduction}

Iodine is an essential micronutrient for the synthesis of thyroid hormones that are critical for brain development and growth during fetal life and infancy. Iodine deficiency during pregnancy may affect cognitive function of the offspring and lead to mental impairment [1-4]. World Health Organization (WHO) considers iodine deficiency to be the single most important preventable cause of brain damage worldwide [5]. Universal salt iodization is the first-line strategy for the elimination of severe iodine deficiency; however, in Norway, no public strategy to ensure adequate iodine status currently exists. Because of successful programs of universal salt iodization in former severely iodine-deficient regions around the world, public health concern has shifted toward mild to moderate iodine deficiency, which remains prevalent in many regions, especially among pregnant women [6]. 
Iodine deficiency is often thought to be a problem in developing countries, however industrialized countries are not immune [7,8]. Indeed, concern is emerging that mild iodine deficiency might be prevalent in Norway, even though Norway has been considered iodine replete for six decades [9]. Results from the Norwegian Mother and Child Cohort Study (MoBa) have shown that a high proportion of pregnant women recruited from 2002 to 2008 had suboptimal iodine intake [10]. A recent study found that lactating women in Norway had low breast milk iodine concentration, and $\frac{3}{4}$ of the women had suboptimal iodine status [11]. In Norway, the most important iodine sources are milk and dairy products, due to iodine fortification of cattle fodder, in addition to seafood. The consumption of milk, yoghurt, and lean fish has declined over the past decades in some population groups. Only one brand of table salt is fortified with iodine and the permitted level $(5 \mu \mathrm{g} / \mathrm{g})$ is too low to affect iodine intake [9]. Iodine in drinking water is low $(<0.2 \mu \mathrm{g} / \mathrm{L})$ [9]. A report on iodine status in Norway highlighted the need for updated studies on iodine status among pregnant women [12]. The main objective in this study was therefore to evaluate iodine status by assessing urinary iodine concentration (UIC) and calculate iodine intake in pregnant women in the Oslo area, Norway. In addition, in a sub-sample, urinary iodine excretion (UIE) from 24-h urine samples was analyzed to compare the agreement between UIC and UIE and calculated iodine intake.

\section{Materials and Methods}

\subsection{Population and Study Design}

Convenience sampling was used to recruit pregnant women from eight different Mother and Child Health Centers (MCHCs) in the Oslo area, Norway. The data were collected in two steps: first, pregnant women were recruited by midwives at MCHCs during antenatal care visits in the period from February to December 2016. All pregnant women who could read and write Norwegian were invited to participate. To assess iodine concentration at population level, at least 500 urine samples are required [13]. Around 50-100 women from each of the eight healthcare centers were asked to participate, in total 804 pregnant women were included. The women answered questions on background information (age, week of pregnancy, previous pregnancies, pre-pregnancy height and weight, educational level and smoking habits, country of birth, years in Norway and languages) and gave a spot urine sample $(n=777)$. In addition, iodine intakes from iodine-rich foods and iodine-containing supplements were calculated for the last $24-\mathrm{h}(n=804)$. Secondly, in a sub-sample of 49 of the 777 pregnant women, urinary iodine excretion was analyzed in 24-h urine, in addition to the spot urine samples. To provide information on habitual intake, they were asked to fill in a food frequency questionnaire with 31 food items in addition to a more in-depth 24-h dietary recall interview covering total food intake on the day of the 24-h urine collection. In total, 777 of 804 women delivered spot urine samples, of which 728 were in the main study and 49 in the sub-study. In total, 804 gave information on iodine intake, of which 755 were in the main study and 49 in the sub-study.

After delivery of urine samples, all women received information sheets about dietary sources of iodine and recommendations for iodine intake. The women were informed about the study purpose and those willing to participate gave informed written consent. The present study was conducted according to the guidelines in the Declaration of Helsinki and was approved by the Regional Committee for Medical and Health Research Ethics Norway (2015/1845).

\subsection{Urinary Iodine Concentration}

Participants donated a non-fasting (random) spot urine sample in a labelled $100 \mathrm{~mL}$ Vacuette ${ }^{\circledR}$ Urine beaker (Greiner Bio-One, Kremsmünster, Austria). Participants in the sub-study were given one 0.5 L and two $1 \mathrm{~L}$ high-density polyethylene plastic bottles (VWR International AS, Oslo, Norway) with wide opening and screw capped double lids for collecting one spot morning urine and 24-h urine. They were instructed to discard the first morning urine void on the day of urine collection, and collect all urine for the next $24 \mathrm{~h}$. The morning urine void on the second day was to be collected 
in a separate container marked "morning". The morning and the 24-h urine samples were stored at $<4{ }^{\circ} \mathrm{C}$ in the time prior to handling. Aliquots of $1.00 \mathrm{~mL}$ morning urine were transferred into $15 \mathrm{~mL}$, polypropylene (pp) centrifuge tube (Sarstedt, Nümbrecht, Germany) by means of a 100-5000 $\mu \mathrm{L}$ electronic pipette (Biohit, Helsinki, Finland) for determination of UIC and the rest added to the 24-h collection. All samples were stored refrigerated from the time of sampling until transportation to the laboratory. At the laboratory, the non-fasting spot urine sample where stored at minus $80{ }^{\circ} \mathrm{C}$ until analysis. Considering both the non-fasting spot urine sample, the morning and the 24-h urine samples, aliquots of $1.00 \mathrm{~mL}$ were diluted ten times with an alkaline solution (BENT), containing $4 \%$ (w/V) 1-Butanol, 0.1\% (w/V) $\mathrm{H}_{4}$ EDTA, 2\% (w/V) $\mathrm{NH}_{4} \mathrm{OH}$, and 0.1\% (w/V) Triton X-100. Reagents of analytical grade or better and deionized water $(>18 \mathrm{M} \Omega)$ were used throughout. The quantification of iodine was performed by means of the Agilent 8800 Triple Quadrupole ICPMS (Agilent, Santa Clara, CA, USA) using oxygen reaction mode. Iodine was determined on mass 127. Tellurium (Te) was used as internal standard. Certified reference materials (CRM) used for quality control was Trace Elements Urine L-1 (78 $\mu \mathrm{g} / \mathrm{L})$ and Trace Elements Urine L-2 $(280 \mu \mathrm{g} / \mathrm{L})$ from Seronorm. All the measured values of CRM were within the certified range. The same procedure was followed for blank samples as other samples, and all had values under the limit of detection (LOD, $0.4 \mu \mathrm{g} / \mathrm{L}$ ) or limit of quantification $(\mathrm{LOQ}, 1.2 \mu \mathrm{g} / \mathrm{L})$. The detection and quantification limits were calculated at three and ten times the standard deviation (SD) of blank samples, respectively. Intermediate precision (within-laboratory reproducibility) was $<4 \%$. The Norwegian University of Life Sciences in Aas (Faculty of Environmental Sciences and Natural Resource Management) performed measurement of the iodine concentration. In sub-study-participants, UIE was calculated by multiplying UIC in the 24-h urine and the 24-h urine volume;

$$
\mathrm{UIE}(\mu \mathrm{g} / 24 \mathrm{~h})=\mathrm{UIC}(\mu \mathrm{g} / \mathrm{L}) \times \text { Total urinary volume }(\mathrm{L} / 24 \mathrm{~h}) .
$$

Estimated iodine intake from UIE was calculated by the assumption that $90 \%$ of dietary iodine is excreted in urine $(\mathrm{UIE} \mu \mathrm{g} / 24 \mathrm{~h} \times 100 / 90)$ [14].

\subsection{Assessment of Iodine from Food and Dietary Supplements}

Iodine intakes from iodine-rich foods and iodine-containing supplements were calculated for the last $24 \mathrm{~h}$. Most participants $(n=755)$ reported intake of iodine-rich foods, while women in the sub-study reported total food intake during the last 24-h and habitual intake (frequency) of selected food items. In the main study, the questionnaire included questions about intake of milk and yoghurt (number of glasses), cheese, eggs (including dishes), and fish (lean and fatty fish for dinner and/or on bread). These are the main food items contributing to iodine intake in Norway [10]. The reported food intakes were multiplied with the iodine concentration for each specified food to obtain iodine amount. In the sub-study $(n=49)$, iodine intake for the last $24 \mathrm{~h}$ was assessed by a 24 -h recall performed as a semi-structured interview. The $24-\mathrm{h}$ recall included all food and drinks consumed, not only iodine-rich foods as in the main study. All food items reported in the 24-h recall were manually coded and FoodCalc [15] was used to calculate food and nutrient intakes. In addition, sub-study participants reported their habitual food intake by answering 31 questions about average intake of selected food items/dishes, including eight questions about iodine-containing foods. Of these, three questions assessed intake of milk and dairy products, four assessed intake of fish and fish-dishes, and one assessed intake of egg and egg-dishes. The questions had seven answer alternatives, ranging from rarely/never to five times daily or more. The answers to the questions related to intake of milk, cheese, fish, and egg were converted to daily amounts and multiplied with the iodine concentration for each food item/dish. In all calculations, iodine concentrations reported in the Norwegian Food Composition Table [16] were used for all items except milk and egg. We applied $13 \mu \mathrm{g} / 100 \mathrm{~g}$ for milk and $30 \mu \mathrm{g} / 100 \mathrm{~g}$ for eggs, as analytical results for iodine concentration in these items were lower than the values in the food composition table [17,18]. For estimating 24-h iodine intake in the main study and the habitual iodine intake in the sub-study, we applied recipes to derive an iodine concentration in composite dishes and for averaging concentrations from different fish species. To account for iodine 
contributed by foods and dishes not covered by the assessed food items, we added $30 \mu \mathrm{g} / \mathrm{day}$ to each estimated total intake. The questionnaire filled in by all participants included a question about use of dietary supplements. Participants were asked to report each supplement by name and how many times weekly the supplement was taken. Using information provided by producers and labels, we calculated the daily amount of iodine contributed by iodine containing supplements and added this to the calculated 24-h and habitual intakes from food.

\subsection{Definitions of Iodine Status and Recommendations for Iodine Intake}

In this study, we apply the epidemiological criteria's for assessment of iodine nutrition established by WHO [5]. The recommended indicator for evaluation of iodine status is the population median UIC, and median UIC below $150 \mu \mathrm{g} / \mathrm{L}$ is considered to reflect insufficient iodine intake in pregnant women, while a median UIC between 150 and 249 reflects adequate iodine nutrition. The Institute of Medicine established an estimated average requirement (EAR) for iodine of $160 \mu \mathrm{g} /$ day during pregnancy [19]. WHO recommends a daily iodine intake of $250 \mu \mathrm{g} /$ day during pregnancy, while the recommended iodine intake in the Nordic countries is set to $175 \mu \mathrm{g} / \mathrm{day}$ during pregnancy [20], and the recommended daily intake for non-pregnant women of reproductive age is similar to the WHO recommendation of $150 \mu \mathrm{g} /$ day [21].

\subsection{Statistical Methods}

Normally distributed data were presented as mean \pm SD. Non-normally distributed data were presented as median and 25th-75th percentiles (p25-p75). UIC, UIE, short-term iodine intake from food and supplements, and habitual iodine intake were checked for normality using Q-Q plots and the Shapiro-Wilk test. Due to the skewed distribution, non-parametric tests were used. Independent group differences were examined using Mann-Whitney U-test and dependent group differences were examined using Wilcoxon's signed rank test. Spearman's rank correlation was used to evaluate the linear relationship between continuous variables. The UIC was used as dependent variable in multiple linear regression analyses. Because of skewed distribution, this dependent variable was log-transformed (lnUIC). All socioeconomic variables and iodine intake were selected to find candidate variables for inclusion in the model. After linear association $(p<0.10)$, the following variables were included in the initial model: maternal age, gestational age, smoking, hypothyroidism and mean daily use of iodine-containing supplement. All covariates showing a linear association $(p<0.10)$ in the crude regression models were included in a preliminary multiple regression model. Excluded variables were reintroduced and those that were still significantly associated in this model $(p<0.10)$ were retained in the final model. Analysis of the residuals was performed to examine the fit of the model.

\section{Results}

Sample characteristics of the pregnant women are presented in Table 1. In the total sample, $53 \%$ of the women were in the third trimester of pregnancy, $77 \%$ were born in Norway and 53\% expected their first child. The proportion of nulliparous women were higher in the sub-study (86\%) than in the main study (52\%). Women in the sub-study were more often highly educated (55\%), had Norwegian origin (84\%) and reported more often use of iodine supplements (45\%) than women in the main study (32\%). The iodine level in the supplements ranged from 150-200 $\mu \mathrm{g}$.

Table 1. Sample characteristics of the pregnant women ${ }^{\mathrm{a}}$.

\begin{tabular}{lccc}
\hline \multicolumn{1}{c}{ Characteristics } & Total Study $(\boldsymbol{n}=\mathbf{8 0 4})$ & Main Study $(\boldsymbol{n = 7 5 5 )}$ & Sub-Study $(\boldsymbol{n}=\mathbf{4 9})$ \\
\hline Age, years & $31.1 \pm 4.4$ & $31.1 \pm 4.5$ & $30.7 \pm 3.5$ \\
\hline Pre-pregnancy BMI, $\mathrm{kg} / \mathrm{m}^{2}$ & $22.8(21.0-25.2)$ & $22.8(20.9-25.3)$ & $22.5(21.2-24.3)$ \\
\hline
\end{tabular}


Table 1. Cont.

\begin{tabular}{|c|c|c|c|}
\hline Characteristics & Total Study $(n=804)$ & Main Study $(n=755)$ & Sub-Study $(n=49)$ \\
\hline \multicolumn{4}{|l|}{ Gestational weeks b } \\
\hline 1st trimester & $28(3.5)$ & $27(3.6)$ & $1(2.0)$ \\
\hline 2nd trimester & $344(42.8)$ & $329(43.6)$ & $15(30.6)$ \\
\hline 3rd trimester & $426(53.0)$ & $393(52.1)$ & $33(67.3)$ \\
\hline \multicolumn{4}{|l|}{ Parity } \\
\hline Nulliparous & $426(53.0)$ & $384(50.9)$ & $42(85.7)$ \\
\hline Primiparous & $296(36.8)$ & $289(38.3)$ & $7(14.3)$ \\
\hline Multiparous & $82(10.2)$ & $82(10.8)$ & 0 \\
\hline \multicolumn{4}{|l|}{ Country of birth } \\
\hline Norway & $620(77.1)$ & $579(76.7)$ & $41(83.7)$ \\
\hline Other & $184(22.9)$ & $176(23.3)$ & $8(16.3)$ \\
\hline \multicolumn{4}{|l|}{ HDI birth country } \\
\hline Very high HDI & $710(88.3)$ & $663(87.8)$ & $46(93.9)$ \\
\hline High HDI & $37(4.6)$ & $36(4.8)$ & $2(4.1)$ \\
\hline Medium HDI & $23(2.9)$ & $23(3.0)$ & 0 \\
\hline Low HDI & $30(3.8)$ & $30(4.0)$ & 0 \\
\hline \multicolumn{4}{|l|}{ Relationship status } \\
\hline Cohabiting & $429(53.4)$ & $393(52.1)$ & $36(73.5)$ \\
\hline Married & $347(43.2)$ & $336(44.5)$ & $11(22.4)$ \\
\hline Single & $19(2.4)$ & $19(2.5)$ & 0 \\
\hline Other & $9(1.1)$ & $7(0.9)$ & $2(4.1)$ \\
\hline \multicolumn{4}{|l|}{ Education } \\
\hline Lower secondary school & $25(3.1)$ & $24(3.2)$ & $1(2.0)$ \\
\hline Higher secondary school & $137(17.0)$ & $133(17.6)$ & $4(8.2)$ \\
\hline$<4$ years of University ${ }^{c}$ & $334(41.5)$ & $317(42.0)$ & $17(34.7)$ \\
\hline$\geq 4$ years of University ${ }^{c}$ & $308(38.3)$ & $281(37.2)$ & $27(55.1)$ \\
\hline \multicolumn{4}{|l|}{ Employment status } \\
\hline Employed & $696(86.6)$ & $653(86.5)$ & $43(87.8)$ \\
\hline Stay at home/Unemployed & $30(3.7)$ & $29(3.8)$ & $1(2.0)$ \\
\hline Student & $38(4.7)$ & $34(4.5)$ & $4(8.2)$ \\
\hline Other & $30(3.7)$ & $29(3.8)$ & $1(2.0)$ \\
\hline Iodine supplement use & $263(32.7)$ & $241(31.9)$ & $22(44.9)$ \\
\hline Smoking during pregnancy & $10(1.2)^{d}$ & $10(1.3)$ & 0 \\
\hline Self-reported use of dry snuff & $10(1.2)^{d}$ & $10(1.3)$ & 0 \\
\hline Thyroid disease (self-reported) & $38(4.7)$ & $34(4.5)$ & $4(8.1)$ \\
\hline Hypothyroidism ${ }^{\mathrm{e}}$ & $32(4.0)$ & $29(3.8)$ & $3(6.1)$ \\
\hline Hyperthyroidism & $6(0.7)$ & $5(0.7)$ & $1(2.0)$ \\
\hline
\end{tabular}

${ }^{a}$ Values given in mean \pm standard deviation (SD), median (p25-p75) and $n(\%)$. In the main study: 10 missing from prior body mass index (BMI), 6 missing from gestational age, 4 missing from human development index (HDI), 10 missing from employment status, and 6 missing from thyroid disease. In the sub-study: 2 missing from HDI; $\mathrm{b}$ 1st trimester $=0-12$ weeks, 2 nd trimester $=13-28$ weeks, 3 rd trimester $=29$ weeks-birth; ${ }^{\mathrm{c}}$ University or University College; ${ }^{\mathrm{d}}$ Daily amounts ranged from $1-10$ cigarettes, both occasionally and daily use; ${ }^{\mathrm{e}}$ Treated with synthetic T4.

Descriptive data of UIC and UIE are presented in Table 2. Median (p25-p75) UIC was 92 (59-140) $\mu \mathrm{g} / \mathrm{L}$. Median (p25-p75) UIC in first, second and third trimester were $92 \mu \mathrm{g} / \mathrm{L}(43-173), 96 \mu \mathrm{g} / \mathrm{L}$ (64-140) and $91 \mu \mathrm{g} / \mathrm{L}$ (58-130), respectively. No significant differences were found in UIC between the trimesters. Median (p25-p75) UIC in the 24-h urine collection was 91 (61-190) $\mu \mathrm{g} / \mathrm{L}$. Median (p24-p75) UIE was $120(83-181) \mu \mathrm{g} / \mathrm{L}$.

As seen from the box plot in Figure 1, the median UIC and upper quartiles were below the recommended level of $150 \mu \mathrm{g} / \mathrm{L}$ in the main study and as well as in the 24-h urine in the sub-study.

Calculated iodine intakes are shown in Table 3. In the main study, the median (p25-p75) 24-h iodine intake from food was $110(70-150) \mu \mathrm{g} /$ day. Including iodine from supplements, the median 
(p25-p75) iodine intake increased to $148(88-251) \mu \mathrm{g} /$ day. In the sub-study, the median (p25-p75) iodine intake by 24 -h recall was 114 (78-149) $\mu \mathrm{g}$ /day from food and 143 (101-289) $\mu \mathrm{g} /$ day including iodine from supplements. The median (p25-p75) habitual iodine intake from food was 117 (95-147) $\mu \mathrm{g} /$ day and 149 (109-268) $\mu \mathrm{g} /$ day including supplemental iodine. The median amount of iodine contributed by supplements was $175 \mu \mathrm{g} /$ day in iodine-containing supplement users in the main study and $150 \mu \mathrm{g} /$ day iodine supplement users in the sub-study.

Table 2. Urinary iodine concentration (UIC) by trimester in spot samples $(n=777)$ a , and UIC, urine volume and urinary iodine excretion (UIE) in $24 \mathrm{~h}$ urine samples in the sub-study $(n=49)$.

\begin{tabular}{cccccc}
\hline & \multicolumn{5}{c}{ UIC $(\boldsymbol{n}=\mathbf{7 7 7})$} \\
\hline UIC $(\mu \mathrm{g} / \mathrm{L})$ & Median & p25-p75 & Mean & SD & Min, Max \\
\hline All trimesters $(n=777)$ & 92 & $59-140$ & 114 & 86 & 11,860 \\
1st Trimester $(n=26)$ & 92 & $43-173$ & 115 & 75 & 23,280 \\
2nd Trimester $(n=332)$ & 96 & $64-140$ & 119 & 94 & 14,860 \\
3rd Trimester $(n=413)$ & 91 & $58-130$ & 110 & 80 & 11,660 \\
\hline & & \multicolumn{5}{c}{ UIE $(\boldsymbol{n}=\mathbf{4 9})$} \\
\hline UIC in 24-h urine $(\mu \mathrm{g} / \mathrm{L})$ & 91 & $61-140$ & 103 & 54 & 24,250 \\
Urine volume $(\mathrm{L} / 24 \mathrm{~h})$ & 1.4 & $1.1-1.8$ & 1.5 & 0.5 & $0.5,3.2$ \\
UIE $(\mu \mathrm{g} / 24 \mathrm{~h})$ & 120 & $83-181$ & 136 & 64 & 43,309 \\
\hline
\end{tabular}

a Six had missing information for gestational age. No significant differences between trimesters $(p=0.381)$, tested by Kruskal-Wallis test.

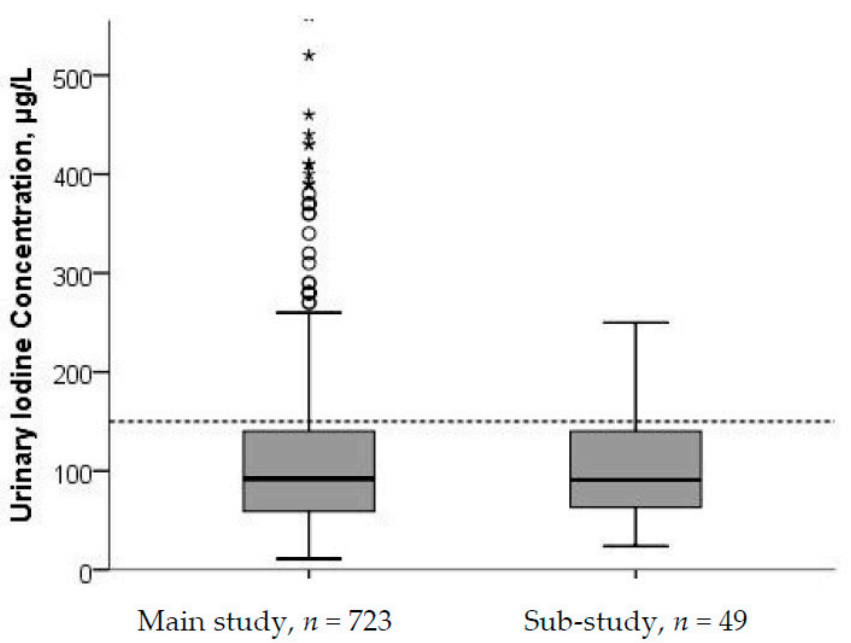

Figure 1. Urinary iodine concentration (UIC) from main study $(n=723)$ and sub-study $(n=49)$. Five cases with UIC $>500 \mu \mathrm{g} / \mathrm{L}$ in the main study were excluded from the figure. The stippled horizontal line marks the epidemiological criteria for assessing adequate iodine nutrition based on median UIC by the WHO [5].

In the main study, $55 \%$ had a calculated iodine intake below EAR (160 $\mu \mathrm{g} /$ day). In the sub-study, $53 \%$ and $55 \%$ had calculated iodine intake below $160 \mu \mathrm{g} /$ day in the 24-h recall interview and habitual intake, respectively. In the total sample, total iodine intake correlated with UIC; $r=0.26$ (95\% confidence interval (CI): 0.19, 0.32). In the sub-sample, total iodine intake correlated with UIE for the 24-h recall $(r=0.46 ; 95 \%$ CI: $0.21,0.66)$ and for habitual food intake $(r=0.36 ; 95 \%$ CI: 0.09, 0.58). Significant, but slightly weaker correlations were found for the calculated intakes and UIC (data not shown). Comparison of dietary iodine intake and iodine estimated from UIC and UIE (Table 3) showed that total iodine intake calculated from the 24-h recall as well as the habitual iodine intake by the FFQ were significantly higher than iodine intake estimated from UIE ( $p=0.17$ and $p=0.02$, respectively). Likewise, iodine intake estimated from UIC was significantly higher than that estimated from UIE $(p<0.01)$. 
Median (p25-p75) UIC among the supplement users was 120 (80-190) $\mu \mathrm{g} / \mathrm{L}$ and 83 (54-120) $\mu \mathrm{g} / \mathrm{L}$ among the non-supplement users.

Table 3. Calculated iodine intake from food and from food and supplements reported from 24-h recall of iodine-rich foods in the main study and habitual intake and 24-h recall in the sub-study.

\begin{tabular}{|c|c|c|c|c|c|}
\hline \multirow[b]{2}{*}{ Iodine Intake ( $\mu \mathrm{g} /$ day) } & \multicolumn{5}{|c|}{ Main Study $(n=755)$} \\
\hline & Median & p25-p75 & Mean & SD & Min, Max \\
\hline 24-h intake from food ${ }^{a}$ & 110 & $70-150$ & 121 & 67 & 30,667 \\
\hline 24-h total intake ${ }^{\mathrm{b}, \S}$ & 148 & $86-251$ & 175 & 105 & 30,689 \\
\hline \multirow[t]{2}{*}{ Estimated intake from UIC } & 145 & 89-214 & 176 & 133 & 16,1183 \\
\hline & \multicolumn{5}{|c|}{ Sub-Study $(n=49)$} \\
\hline 24-h intake from food & 114 & $78-149$ & 128 & 75 & 18,403 \\
\hline 24-h total intake ${ }^{c, \S \S}$ & 143 & $101-289$ & 188 & 106 & 18,403 \\
\hline Habitual intake from food & 117 & $95-147$ & 122 & 37 & 50,206 \\
\hline Habitual total intake $\mathrm{d}, \S \S$ & 149 & $109-268$ & 182 & 90 & 56,361 \\
\hline Estimated intake from UIC ${ }^{\mathrm{e}}$ & 157 & $103-257$ & 193 & 122 & 103,257 \\
\hline Estimated intake from UIE ${ }^{\mathrm{e}, \mathrm{f}}$ & 133 & 92-201 & 151 & 71 & 48,343 \\
\hline
\end{tabular}

Differences tested with Wilcoxon Signed Ranks test; ${ }^{\mathrm{a}}$ Iodine intake from iodine-rich foods; ${ }^{\mathrm{b}}$ Iodine intake from iodine-rich foods and supplements; ${ }^{\mathrm{c}}$ Iodine intake from food and supplements; ${ }^{\mathrm{d}}$ Iodine intake from food and supplements; ${ }^{\mathrm{e}}$ Estimated iodine intake from UIC $=$ UIC $(\mu \mathrm{g} / \mathrm{L}) \times 0.0235 \times$ bodyweight $(\mathrm{kg})$ [19]; ${ }^{\mathrm{f}}$ Estimated iodine intake from UIE given 90\% excretion of ingested iodine = UIE $(\mu \mathrm{g} / 24-\mathrm{h}) \times 100 / 90 ; \S$ Total 24-h iodine intake did not differ from iodine intake estimated from UIC ( $p=0.2$; Wilcoxon's signed rank test); $\$ \S$ Total 24-h iodine intake, total habitual iodine intake, and iodine intake estimated from UIC were all significantly higher than iodine intake estimated from UIE ( $p<0.05$; Wilcoxon's signed rank test).

Median (p25-p75) UIE among the supplement users was $165(100-223) \mu g / 24-h$ and 105 (77-187) $\mu \mathrm{g} / 24-\mathrm{h}$ among the non-supplement users. Participants who reported use of iodine-containing supplements had higher UIC $(p<0.001)$ than non-supplement users, and, in the sub-study, this was evident also for UIE ( $p=0.021$ ) (Figures 2 and 3). For UIC, the median values were below the cut-off from WHO of $150 \mu \mathrm{g} / \mathrm{L}$ both among supplement and non-supplement users. In the sub-study, data from the 24-h recall interview showed that the main dietary iodine sources were milk and dairy products, contributing with on average $55 \%$ of the daily iodine intake, and seafood contributing with on average $18 \%$ of the daily iodine intake.

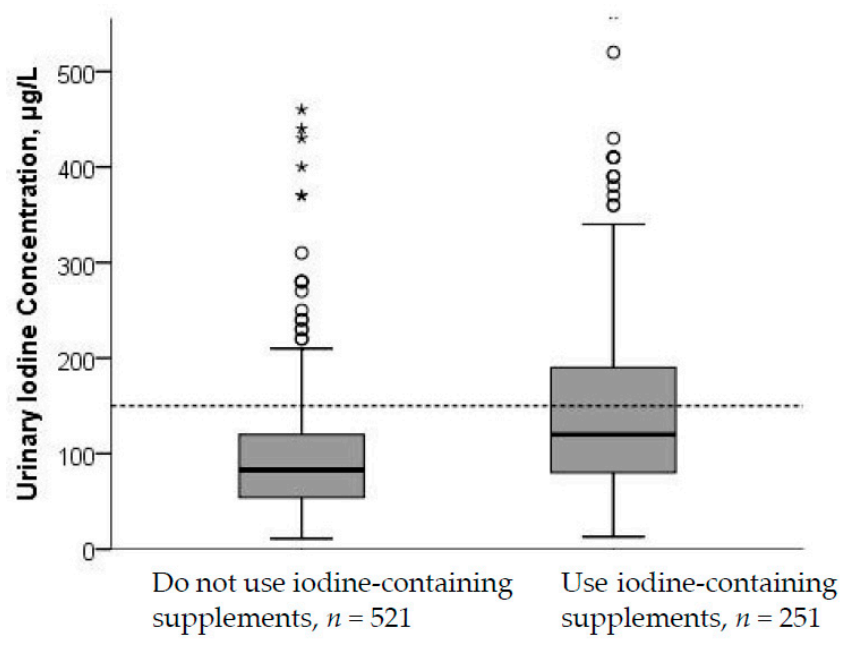

Figure 2. UIC by use of iodine-containing supplements. Five cases with UIC $>500 \mu \mathrm{g} / \mathrm{L}$ were excluded from the figure. The difference in UIC between non-supplement and supplement users was significant $(p<0.001$; Mann-Whitney U test). The stippled horizontal line marks the epidemiological criteria for assessing adequate iodine nutrition based on median UIC by the WHO [5]. 


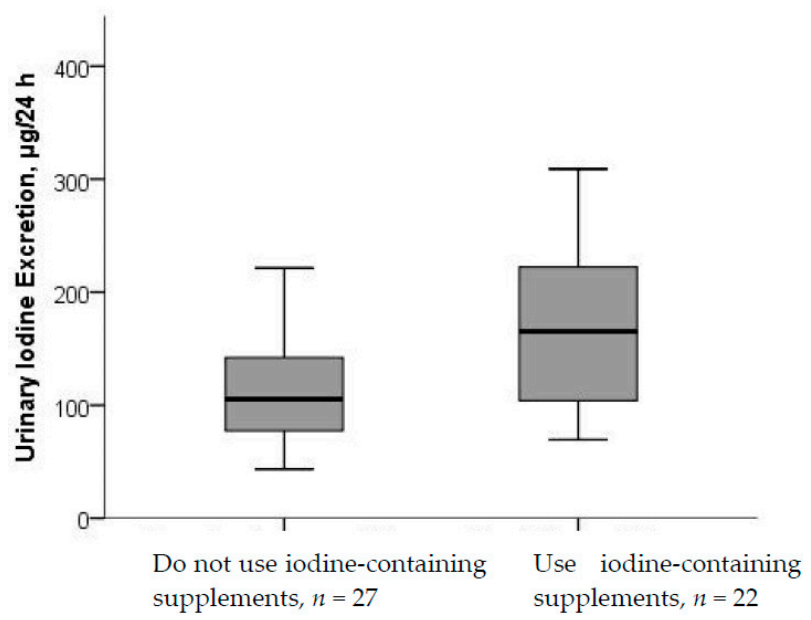

Figure 3. UIE by use of iodine-containing supplements. Differences in UIE between non-supplement and supplement users were significant ( $p<0.021$; Mann-Whitney U test).

Predictors of UIC are shown in Table 4. Maternal age, hypothyroidism, and use of iodine-containing supplements were associated with increased UIC, while gestational weeks and smoking during pregnancy were associated with decreased UIC. These predictors explained $10.5 \%$ of the variance in UIC.

Table 4. Predictors of urinary iodine concentration (UIC) in pregnant women in Oslo $(n=777)$, with $\mathrm{UIC}^{\mathrm{a}}(\mu \mathrm{g} / \mathrm{L})$ as dependent variable.

\begin{tabular}{|c|c|c|c|c|c|}
\hline Predictor Variables & $\begin{array}{l}\text { Unadjusted Coefficient } \\
(95 \% \mathrm{CI})\end{array}$ & $p$ & $\begin{array}{c}\text { Adjusted Coefficient } \\
(95 \% \mathrm{CI})\end{array}$ & $p$ & Stand Beta \\
\hline Constant & & & $4.181(3.849,4.513)$ & $<0.001$ & \\
\hline Maternal age & $0.015(0.005,0.025)$ & 0.004 & $0.013(0.003,0.022)$ & 0.011 & 0.088 \\
\hline Gestational weeks & $-0.004(-0.010,0.001)$ & 0.139 & $-0.007(-0.012,-0.001)$ & 0.019 & -0.081 \\
\hline Hypothyroidism $^{b}$ & $0.291(0.057,0.525)$ & 0.015 & $0.313(0.090,0.536)$ & $<0.001$ & 0.289 \\
\hline 24-h iodine Suppl ${ }^{\mathrm{c}}$ & $0.002(0.001,0.002)$ & $<0.001$ & $0.002(0.002,0.003)$ & 0.006 & 0.094 \\
\hline Smoking $\mathrm{d}$ & $-0.529(-0.951,-0.108)$ & 0.014 & $-0.442(-0.869,-0.016)$ & 0.042 & -0.070 \\
\hline$R^{2}$ & & & & 0.105 & \\
\hline
\end{tabular}

${ }^{a}$ UIC log transformed, 6 missing from gestational age and hypothyroidism, and 10 missing in the adjusted model due to missing cases in independent the variables mentioned, $\left(95 \% \mathrm{CI}=95 \%\right.$ confidence interval); ${ }^{\mathrm{b}}$ Self-reported hypothyroidism $\left(0=\right.$ no, $1=$ yes); ${ }^{\mathrm{c}}$ Mean daily intake of iodine from supplements (habitual intake); ${ }^{\mathrm{d}}$ Categories for smoking during pregnancy $(0=$ no, $1=$ yes $)$.

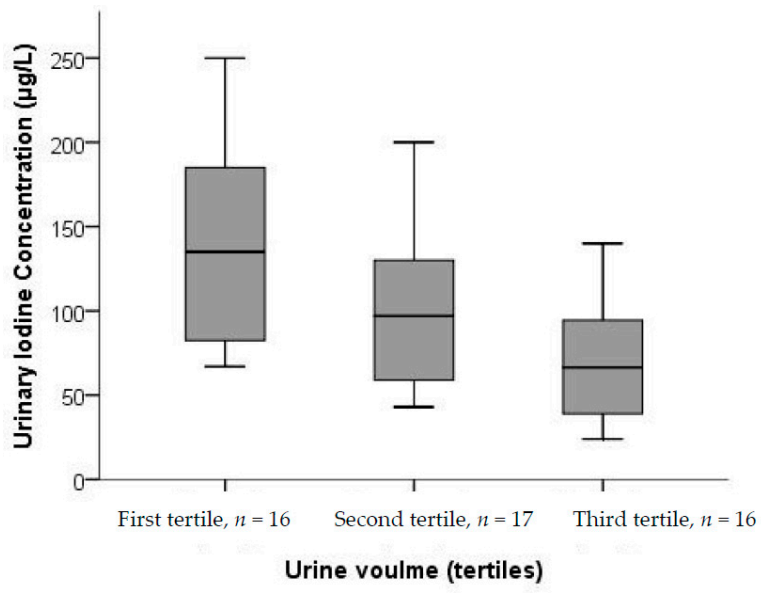

Figure 4. UIC from spot samples in 24-h urine samples by tertiles of total excreted urine volume $(n=49)$. There were significant differences in UIC between the tertiles ( $p=0.002 ;$ Kruskal-Wallis test). 
Hydration status is known to influence UIC and in the sub-study we examined UIC by increasing 24-h urine volume and found that UIC in the 24-h urine decreased as urine volume increased (Figure 4). The decrease in UIC by urine volume was significant $(p=0.002)$.

\section{Discussion}

A large proportion of the pregnant women in the Oslo area were mild to moderately iodine deficient. This finding was evident from evaluation of urinary iodine as well as from calculated iodine intake. In utero, severe iodine deficiency causes irreversible damage to the developing brain [6], while the impact of mild to moderate iodine deficiency is less clear. There are indications, however, that mildly decreased maternal thyroid function in pregnancy may result in cognitive delays in the offspring [1-3,22-24]. These studies show the importance of adequate iodine status during early gestation and emphasize the risk that iodine deficiency can pose to the developing infant, even in countries classified as only mildly iodine deficient.

Although there is no national screening program for measuring iodine status, Norway has been considered iodine replete for six decades $[9,10]$. Data from a recent study on iodine status in the Nordic countries found that pregnant and lactating women might be mildly iodine deficient [9]. In the present study, we found a median UIC of $92 \mu \mathrm{g} / \mathrm{L}$, which is in accordance to previous findings on iodine status among pregnant women in Norway. A Norwegian study in 1008 pregnant women recruited from 2011 to 2012 found that $80 \%$ of the pregnant women had UIC below $150 \mu \mathrm{g} / \mathrm{L}$, with median UIC of $82 \mu \mathrm{g} / \mathrm{L}$ [25]. In the Northern Norway Mother-and-Child Contaminant Cohort Study (MISA), iodine status was measured in 197 pregnant women recruited from 2007 to 2009. A median UIC of $84 \mu \mathrm{g} / \mathrm{L}$ was found and $80 \%$ had UIC $<150 \mu \mathrm{g} / \mathrm{L}$ [26]. In the Norwegian Mother and Child Cohort Study (MoBa), 119 participants recruited for a validation study from 2003 to 2004 collected 24-h urine samples. Median UIC was $69 \mu \mathrm{g} / \mathrm{L}$ and $89 \%$ had UIC < $150 \mu \mathrm{g} / \mathrm{L}$ [10]. A Norwegian study in 63 non-pregnant women recruited from 1999 to 2001 reported a median UIC of $112 \mu \mathrm{g} / \mathrm{L}$ in Northern Norway and $82 \mu \mathrm{g} / \mathrm{L}$ in Western Norway [27]. According to our knowledge, the present study is the largest and has the most recent data on UIC in Norwegian women (recruited in 2016) and the results add to the evidence that a large proportion of pregnant women have insufficient iodine intake and that iodine nutrition is a public health concern in Norway. Inadequate iodine status has also been reported in other Nordic countries. A cross-sectional study in Denmark from 2012 in 158 pregnant found a median UIC of $119 \mu \mathrm{g} / \mathrm{L}$ [28]. The median UIC was $130 \mu \mathrm{g} / \mathrm{L}$ in iodine-containing supplement users and $76 \mu \mathrm{g} / \mathrm{L}$ in non-supplement users. The median UIC in a Swedish cross-sectional study in 459 pregnant women with urine sampled from 2010 to 2012 was $98 \mu \mathrm{g} / \mathrm{L}$ [29].

In the current study, approximately half of the women had a total iodine intake below $160 \mu \mathrm{g} /$ day, which is the EAR for pregnant women. This strengthens the finding of insufficient iodine intake reflected by low UIC and confirm high probability of iodine inadequacy in this population. Results from the sub-study, in which urinary iodine was measured in 24-h urine samples and participants provided a detailed dietary recall, confirmed the insufficient iodine status observed in the main study. Calculated iodine intake in more than 60,000 pregnant women in MoBa recruited from 2002 to 2008 showed that more than $50 \%$ had iodine intake lower than the Nordic recommendations of $175 \mu \mathrm{g} /$ day [10], and 74\% had an estimated intake from food lower than the EAR [1]. Another study in Norwegian pregnant women recruited from 2011 to $2012(n=833)$ reported a median iodine intake of $153 \mu \mathrm{g} /$ day from food and supplements. Use of iodine-containing supplements was reported by $14 \%$ of the participants [25]. An increasing number of Norwegians take dietary supplements, and today many multivitamin-mineral supplements contain iodine [9]. The Norwegian guidelines to pregnant women was recently updated to include a recommendation to use of iodine containing supplements (150 $\mu \mathrm{g} /$ day) for women with no or low intake of milk [30].

In the present study, $33 \%$ of all women reported use iodine-containing supplements, which on average contributed with $175 \mu \mathrm{g} /$ day to these women's diet. The median UIC was significantly higher in supplement users than in non-supplement users; $120 \mu \mathrm{g} /$ day and $83 \mu \mathrm{g} /$ day, respectively. 
Similarly, in the MoBa Study, 32\% of the pregnant women took iodine-containing supplements which on average contributed with $100 \mu \mathrm{g} /$ day to these women's diet [10]. Maternal iodine supplementation in areas of mild-to-moderate iodine deficiency may improve cognitive performance of the offspring, but randomized controlled studies with long-term outcomes are lacking [6].

The iodine requirement increases during pregnancy because of a rise in maternal T4 production to maintain maternal euthyroidism and transfer of thyroid hormones to the fetus, iodine-transfer to the fetus, particularly in late gestation, and a likely increase in renal iodine clearance [31,32]. In Norway, there are few dietary sources of iodine [33] and the individual intake of these food groups varies significantly. In addition, the consumption of seafood, especially lean fish, and milk has declined, especially in the female population [10,34]. In this present study, food groups that contributed most to the iodine intake were milk and milk products $(55 \%)$ followed by seafood $(18 \%)$. Even though the participants had a mean daily intake of milk/yoghurt of 3-4 dL, and the majority reported regular consumption of fish, the food sources alone did not provide the amounts of iodine required to meet maternal and fetal needs during pregnancy. In Norway, pregnant women who do not consume or have low intake of dairy and/or seafood, and who do not obtain iodine from supplements are at great risk of inadequate iodine intakes [10,12].

In the multiple regression analysis iodine-containing supplements was the strongest predictor for UIC. The result confirms that iodine-containing supplement use is an important determinant of iodine status and an important iodine source for pregnant women. Maternal age was positively associated with UIC, which has also been found in another study [35]. A potential explanation could be that older women consume more iodine-rich foods [36]. However, this has not been confirmed in Norway. Suffering from hypothyroidism was a strong positive predictor for UIC. Women with hypothyroidism are prescribed a synthetic thyroid hormone (levothyroxine), with iodine, which explains the positive association between hypothyroidism and UIC. A negative borderline significant association between gestational age and UIC was found. One explanation could be an increased iodine transfer to the fetus throughout pregnancy. However, this result is not consistently found in other studies. In a cross-sectional study of pregnant women, UIC increased with gestational age, but the differences between the first, second and third trimester were not statistically significant [37]. In an Austrian study of 246 pregnant women, neither maternal nor gestational age influenced UIC (median UIC: $87 \mu \mathrm{g} / \mathrm{L}$ ) [38]. Median UIC in pregnant women in Tasmania declined after elevated excretion of iodine seen in early pregnancy [39]. We found that smoking was negatively associated with UIC. Lower UIC in smokers than in non-smokers was also found in another study in Norway [40]. Tobacco smoke contains thiocyanate, a chemical toxin that accumulates in blood and tissues and has goitrogenic properties [41], but this does not explain why smoking results in lower excretion of iodine. A possible reason for the inverse relationship between smoking and UIC could be related to differences in dietary pattern between smokers and non-smokers. Our results showed no significant association between ethnicity and UIC, contrary to other studies [38,42].

In the sub-study, estimated iodine intake from UIE (133 $\mu \mathrm{g} /$ day) were lower than the calculated iodine intakes by in $24-\mathrm{h}$ recall $(143 \mu \mathrm{g} /$ day $)$ and habitual intake ( $149 \mu \mathrm{g} /$ day). This might be due to over reporting of food and supplement use or that food iodine concentrations were lower than the values applied in the calculation. Comparison of UIC and urine volume confirmed that hydration status influenced UIC $[13,43]$ and showed that UIE is a more reliable marker of iodine status at the individual level than UIC. However, both habitual iodine intake, 24-h iodine intake and UIC in the 24-h urine correlated with UIE, indicating good conformity between the methods. Collecting 24-h urine samples is a large participant-burden. Furthermore, several 24-h urine collections are needed in order to assess reliable iodine status at the individual level and urine collection is subjected to bias introduced by failure to collect total volume [44]. Results from the sub-study, for which urinary iodine was measured in 24-h urine and who provided a detailed dietary recall as well as habitual food intake, confirmed the insufficient iodine status observed in the main study. 
The major strength of this study is the relatively large sample of pregnant women $(n=804)$ from different parts of Oslo, including $23 \%$ with an ethnicity other than Norwegian. UIC from at least 500 individuals are required to define iodine status in a population $[8,13]$. Secondly, in the sub-study, we assessed both 24-h urinary iodine excretion and collected detailed information on total 24-h and habitual iodine intake. A major weakness of this study is that the women were not randomly selected and therefore the findings cannot be generalized to all pregnant women in Norway. On the other hand, the current study support findings from other studies in other parts of Norway, including the large MoBa study. Selection bias is a concern also in MoBa [45], where the women were better educated and included fewer smokers than the general population of pregnant women. The proportion of highly educated women ( $\geq 4$ years of University) was significantly higher in our study $(40 \%)$ than in the rest of the female Norwegian population ( $8 \%$ ). Higher education is associated with healthier diet and lifestyle. Despite the high level of education among the participants, insufficient iodine status was evident from both UIC and calculated iodine intakes. The results highlight the often neglected but critical need for routine, periodic monitoring of iodine status in representative population samples, and that national surveys should monitor not only iodine concentrations in school age children, but also include pregnant women as an emerging literature shows that pregnant women may be deficient even where the general population has adequate intake. This implies that iodine insufficiency is present in a larger scale than expected, and it is important the National Health Authorities implement actions immediately. Another limitation of the present study is that a single iodine value was applied to all iodine rich food items (e.g., $130 \mu \mathrm{g} / \mathrm{L}$ ) for milk and yoghurt, while the true content is likely subject to seasonal and other variation

\section{Conclusions}

In conclusion, pregnant women in the Oslo area are mild to moderately iodine deficient. Median UIC and UIE in the sub-study confirmed the finding of insufficient iodine intake in the main study. Approximately half of the women had a total iodine intake below $160 \mu \mathrm{g} /$ day, which is the EAR for pregnant women. Iodine-containing supplements were the strongest predictor for UIC and an important iodine source for pregnant women, however only one-third reported use of iodine-containing supplements. Comparison of UIC and UIE showed that hydration status influenced UIC and that UIE is a more reliable biomarker for iodine status at the individual level than UIC. In Norway, even with an intake according to the national food-based recommendations, the iodine requirement during pregnancy will not be meet by food only. The current study results underline the importance of implementing actions to improve iodine nutrition in Norway.

Acknowledgments: We thank the health professionals at the Mother and Health Centers who recruited participants for this study. TINE AS, a Norwegian dairy company, has partly funded this study.

Author Contributions: S.H., I.A., E.B., Z.P. and A.L.B. planned the study. S.H., I.A., A.M.L., S.B. and L.G.-H. were involved in the data collection. E.L.F.G. was responsible for the urinary iodine analysis. A.L.B. and S.B. calculated iodine intakes. S.H. and I.A. performed the statistical analysis. S.H. drafted the manuscript. All authors contributed to the interpretation of the results and contributed to writing of the manuscript.

Conflicts of Interest: The authors declare no conflict of interest. The founding sponsors had no role in the design of the study, in the collection, analyses, or interpretation of data; in the writing of the manuscript, and in the decision to publish the results.

\section{References}

1. Abel, M.H.; Caspersen, I.H.; Meltzer, H.M.; Haugen, M.; Brandlistuen, R.E.; Aase, H.; Alexander, J.; Torheim, L.E.; Brantsaeter, A.L. Suboptimal Maternal Iodine Intake Is Associated with Impaired Child Neurodevelopment at 3 Years of Age in the Norwegian Mother and Child Cohort Study. J. Nutr. 2017, 147, 1314-1324. [CrossRef] [PubMed]

2. Bath, S.C.; Steer, C.D.; Golding, J.; Emmett, P.; Rayman, M.P. Effect of inadequate iodine status in UK pregnant women on cognitive outcomes in their children: Results from the Avon Longitudinal Study of Parents and Children (ALSPAC). Lancet 2013, 382, 331-337. [CrossRef] 
3. Hynes, K.L.; Otahal, P.; Hay, I.; Burgess, J.R. Mild iodine deficiency during pregnancy is associated with reduced educational outcomes in the offspring: 9-year follow-up of the gestational iodine cohort. J. Clin. Endocrinol. Metab. 2013, 98, 1954-1962. [CrossRef] [PubMed]

4. Taylor, P.N.; Okosieme, O.E.; Dayan, C.M.; Lazarus, J.H. Therapy of endocrine disease: Impact of iodine supplementation in mild-to-moderate iodine deficiency: Systematic review and meta-analysis. Eur. J. Endocrinol. 2014, 170, R1-R15. [CrossRef] [PubMed]

5. World Health Organization (WHO); The United Nations Children's Fund (UNICEF); International Council for Control of Iodine Deficiency Disorders (ICCIDD). Assessment of Iodine Deficiency Disorders and Monitoring Their Elimination; WHO: Geneva, Switzerland, 2007.

6. Pearce, E.N.; Lazarus, J.H.; Moreno-Reyes, R.; Zimmermann, M.B. Consequences of iodine deficiency and excess in pregnant women: An overview of current knowns and unknowns. Am. J. Clin. Nutr. 2016, 104 (Suppl. 3), S918-S923. [CrossRef] [PubMed]

7. Andersson, M.; Karumbunathan, V.; Zimmermann, M.B. Global iodine status in 2011 and trends over the past decade. J. Nutr. 2012, 142, 744-750. [CrossRef] [PubMed]

8. Gizak, M.; Gorstein, J.; Andersson, M. Epidemiology of Iodine Deficiency in Iodine Deficiency Disorders and Their Elimination; Pearce, E.N., Ed.; Springer: Cham, Switzerland, 2017.

9. Nystrom, H.F.; Brantsaeter, A.L.; Erlund, I.; Gunnarsdottir, I.; Hulthen, L.; Laurberg, P.; Mattisson, I.; Rasmussen, L.B.; Virtanen, S.; Meltzer, H.M. Iodine status in the Nordic countries-Past and present. Food Nutr. Res. 2016, 60, 31969. [CrossRef] [PubMed]

10. Brantsaeter, A.L.; Abel, M.H.; Haugen, M.; Meltzer, H.M. Risk of suboptimal iodine intake in pregnant Norwegian women. Nutrients 2013, 5, 424-440. [CrossRef] [PubMed]

11. Henjum, S.; Lilleengen, A.M.; Aakre, I.; Dudareva, A.; Gjengedal, E.L.F.; Meltzer, H.M.; Brantsaeter, A.L. Suboptimal Iodine Concentration in Breastmilk and Inadequate Iodine Intake among Lactating Women in Norway. Nutrients 2017, 9, 643. [CrossRef] [PubMed]

12. National Nutrition Council. The Risk of Iodine Deficeincy in Norway. Identification of an Acute Need for Action, in Norwegian; Technical Report; The Norwegian Directorate of Health: Oslo, Norway, 2016.

13. Vejbjerg, P.; Knudsen, N.; Perrild, H.; Laurberg, P.; Carle, A.; Pedersen, I.B.; Rasmussen, L.B.; Ovesen, L.; Jorgensen, T. Thyroglobulin as a marker of iodine nutrition status in the general population. Eur. J. Endocrinol. 2009, 161, 475-481. [CrossRef] [PubMed]

14. Zimmermann, M.B.; Andersson, M. Assessment of iodine nutrition in populations: Past, present, and future. Nutr. Rev. 2012, 70, 553-570. [CrossRef] [PubMed]

15. Lauritsen, J.; FoodCalc. Data Program from the Project "Diet, Cancer and Health" at the Danish Cancer Society, Denmark. Available online: http:/ /ibt.ku.dk/jesper/foodcalc (accessed on 1 February 2005).

16. Norwegian Food Safety Authority. The Norwegian Food Composition Table; The Norwegian Directorate of Health and University of Oslo: Oslo, Norway, 2016.

17. Troan, G.; Dahl, L.; Meltzer, H.M.; Abel, M.H.; Indahl, U.G.; Haug, A.; Prestlokken, E. A model to secure a stable iodine concentration in milk. Food Nutr. Res. 2015, 59, 29829. [CrossRef] [PubMed]

18. Kielland, E.; Dalane, J.Ø.; Håland, J.T.; Tharaldsen, A. Analyses of Eggs and Chicken, Nutrients and Environmental Contaminants, in Norwegian; The Norwegian Food Safety Authority: Oslo, Norway, 2016.

19. Institute of Medicine. Dietary Reference Intakes for Vitamin A, Vitamin K, Arsenic, Boron, Chromium, Copper, Iodine, Iron, Manganese, Molybdenum, Nickel, Silicon, Vanadium, and Zinc; Institute of Medicine: Washington, DC, USA, 2001.

20. Nordic Council of Ministers. Nordic Nutrition Recommendations; Nordic Council of Ministers: Copenhagen, Denmark, 2012.

21. WHO Secretariat; Andersson, M.; de Benoist, B.; Delange, F.; Zupan, J. Prevention and control of iodine deficiency in pregnant and lactating women and in children less than 2-years-old: Conclusions and recommendations of the Technical Consultation. Public Health Nutr. 2007, 10, 1606-1611. [CrossRef] [PubMed]

22. Skeaff, S.A. Iodine deficiency in pregnancy: The effect on neurodevelopment in the child. Nutrients 2011, 3, 265-273. [CrossRef] [PubMed]

23. Zimmermann, M.B. The Importance of Adequate Iodine during Pregnancy and Infancy. World Rev. Nutr. Diet. 2016, 115, 118-124. [CrossRef] [PubMed] 
24. Trumpff, C.; De Schepper, J.; Tafforeau, J.; Van Oyen, H.; Vanderfaeillie, J.; Vandevijvere, S. Mild iodine deficiency in pregnancy in Europe and its consequences for cognitive and psychomotor development of children: A review. J. Trace Elem. Med. Biol. 2013, 27, 174-183. [CrossRef] [PubMed]

25. Sanchez, P.V.R. Urinary Iodine Concentration and Iodine Intake in Pregnant Norwegian Women. Results from the "Little in Norway" Study (LiN). Master's Thesis, University of Bergen, Bergen, Norway, 2015.

26. Berg, V.; Nost, T.H.; Skeie, G.; Thomassen, Y.; Berlinger, B.; Veyhe, A.S.; Jorde, R.; Odland, J.O.; Hansen, S. Thyroid homeostasis in mother-child pairs in relation to maternal iodine status: The MISA study. Eur. J. Clin. Nutr. 2017, 71, 1002-1007. [CrossRef] [PubMed]

27. Dahl, L.; Meltzer, H.M.; Opsahl, J.A.; Julshamn, K. Iodine intake and status in two groups of Norwegians. Scand. J. Nutr. 2003, 47, 170-178. [CrossRef]

28. Andersen, S.L.; Sorensen, L.K.; Krejbjerg, A.; Moller, M.; Laurberg, P. Challenges in the evaluation of urinary iodine status in pregnancy: The importance of iodine supplement intake and time of sampling. Eur. Thyroid J. 2014, 3, 179-188. [CrossRef] [PubMed]

29. Granfors, M.; Andersson, M.; Stinca, S.; Akerud, H.; Skalkidou, A.; Poromaa, I.S.; Wikstrom, A.K.; Nystrom, H.F. Iodine deficiency in a study population of pregnant women in Sweden. Acta Obstet. Gynecol. Scand. 2015, 94, 1168-1174. [CrossRef] [PubMed]

30. The Norwegian Directorate of Health. Dietary Intake for Pregnant Women 2018. Available online: https:/ / helsedirektoratet.no/folkehelse / graviditet-fodsel-og-barsel/graviditet-og-svangerskap/ kosthold-for-gravide (accessed on 18 February 2018).

31. Stagnaro-Green, A.; Sullivan, S.; Pearce, E.N. Iodine supplementation during pregnancy and lactation. JAMA 2012, 308, 2463-2464. [CrossRef] [PubMed]

32. Pearce, E.N. Effects of iodine deficiency in pregnancy. J. Trace Elem. Med. Biol. 2012, 26, 131-133. [CrossRef] [PubMed]

33. Dahl, L.; Johansson, L.; Julshamn, K.; Meltzer, H.M. The iodine content of Norwegian foods and diets. Public Health Nutr. 2004, 7, 569-576. [CrossRef] [PubMed]

34. Gunnarsdottir, I.; Dahl, L. Iodine intake in human nutrition: A systematic literature review. Food Nutr. Res. 2012, 56. [CrossRef] [PubMed]

35. Bath, S.C.; Walter, A.; Taylor, A.; Wright, J.; Rayman, M.P. Iodine deficiency in pregnant women living in the South East of the UK: The influence of diet and nutritional supplements on iodine status. Br. J. Nutr. 2014, 111, 1622-1631. [CrossRef] [PubMed]

36. Henderson, L.I.K.; Gregory, J.; Bates, C.J.; Prentice, A.; Perks, J.; Swan, G.; Farron, M. National Diet and Nutrition Survey: Adults Aged 19 to 64 Years. Vitamin and Mineral Intake and Urinary Analytes; The Stationery Office: London, UK, 2003.

37. Grewal, E.; Khadgawat, R.; Gupta, N.; Desai, A.; Tandon, N. Assessment of iodine nutrition in pregnant north Indian subjects in three trimesters. Indian J. Endocrinol. Metab. 2013, 17, 289-293. [CrossRef] [PubMed]

38. Lindorfer, H.; Krebs, M.; Kautzky-Willer, A.; Bancher-Todesca, D.; Sager, M.; Gessl, A. Iodine deficiency in pregnant women in Austria. Eur. J. Clin. Nutr. 2015, 69, 349-354. [CrossRef] [PubMed]

39. Stilwell, G.; Reynolds, P.J.; Parameswaran, V.; Blizzard, L.; Greenaway, T.M.; Burgess, J.R. The influence of gestational stage on urinary iodine excretion in pregnancy. J. Clin. Endocrinol. Metab. 2008, 93, 1737-1742. [CrossRef] [PubMed]

40. Brantsæter, A.L.; Knutsen, H.K.; Johansen, N.C.; Nyheim, K.A.; Erlund, I.; Meltzer, H.M.; Henjum, S. Inadequate Iodine Intake in Population Groups Defined by Age, Life Stage and Vegetarian Dietary Practice in a Norwegian Convenience Sample. Nutrients 2018, 10, 230. [CrossRef]

41. Wiersinga, W.M. Smoking and thyroid. Clin Endocrinol. 2013, 79, 145-151. [CrossRef] [PubMed]

42. Hamrosi, M.A.; Wallace, E.M.; Riley, M.D. Iodine status in pregnant women living in Melbourne differs by ethnic group. Asia Pac. J. Clin. Nutr. 2005, 14, 27-31. [PubMed]

43. Konig, F.; Andersson, M.; Hotz, K.; Aeberli, I.; Zimmermann, M.B. Ten repeat collections for urinary iodine from spot samples or 24-h samples are needed to reliably estimate individual iodine status in women. J. Nutr. 2011, 141, 2049-2054. [CrossRef] [PubMed] 
44. Knudsen, N.; Christiansen, E.; Brandt-Christensen, M.; Nygaard, B.; Perrild, H. Age- and sex-adjusted iodine/creatinine ratio. A new standard in epidemiological surveys? Evaluation of three different estimates of iodine excretion based on casual urine samples and comparison to $24 \mathrm{~h}$ values. Eur. J. Clin. Nutr. 2000, 54, 361-363. [CrossRef] [PubMed]

45. Nilsen, R.M.; Vollset, S.E.; Gjessing, H.K.; Skjaerven, R.; Melve, K.K.; Schreuder, P.; Alsaker, E.R.; Haug, K.; Daltveit, A.K.; Magnus, P. Self-selection and bias in a large prospective pregnancy cohort in Norway. Paediatr. Perinat. Epidemiol. 2009, 23, 597-608. [CrossRef] [PubMed] 